\title{
On the Invariant Regularization in Relativistic Quantum Theory
}

\author{
W. Pauli and F. Villars \\ Swiss Federal Institute of Technology, Zurich, Switzerland
}

(Received May 10, 1949)

\begin{abstract}
The formal method of regularization of mathematical expressions of sums of products of different types of $\delta$-functions is first applied to the example of vacuum polarization. It is emphasized that only a regularization of the whole expression without factorization leads to gauge invariant results. It is further shown, that for the regularization of the expression for the magnetic moment of the electron, a single auxiliary mass is sufficient, provided that different functions of the same particle (e.g., the photon functions $\bar{D}$ and $\left.D^{(1)}\right)$ are regularized in the same way and that the regularization of products of two electron functions is never factorized. The result is then the same as that of using Schwinger's method of introducing suitable parameters as new integration variables in the argument of $\delta$-functions, without using any auxiliary masses.
\end{abstract}

\section{§1. INTRODUCTION}

$\mathbf{I}^{\mathrm{N}}$ spite of many successes of the new relativistically invariant formalism of quantum electrodynamics, ${ }^{1}$ which is based on the idea of "renormalization" of mass and charge, there are still some problems of uniqueness left, which need further clarification. The most important one seems to us to be the problem of the self-energy of the photon, which was raised by Wentzel's2 remark that the formal application of Schwinger's original technique of integration to the resulting integral gives a finite result different from zero for this self-energy. This problem is formally contained in the more general problem of the gauge invariance for the resulting current due to vacuum-polarization by an arbitrary external field (not necessarily by a light wave). Schwinger ${ }^{3}$ has shown that this current is given by

$$
\left\langle j_{\mu}(x)\right\rangle=\frac{i}{2} \int d^{4} x^{\prime}\left\langle\left[j_{\mu}(x), j_{\nu}\left(x^{\prime}\right)\right]\right\rangle_{0} \epsilon\left(x-x^{\prime}\right) A_{\nu}{ }^{\mathrm{ext}}\left(x^{\prime}\right)
$$

when $\epsilon(x)= \pm 1$ for $t \geqq 0 ; A_{\nu}{ }^{{ }^{e x t}}(x)$ is the vector potential of the external field and $\left\langle\left[j_{\mu}(x), j_{\nu}\left(x^{\prime}\right)\right]\right\rangle_{0}$ is the vacuum expectation value of the commulator of $j_{\mu}(x)$ with $j_{\nu}\left(x^{\prime}\right)$. The condition for the gauge invariance of this expression for $\left\langle j_{\mu}(x)\right\rangle$ (which includes the vanishing of the photon self-energy as a special case) is:

$$
\partial / \partial x_{\mu}\left\{\left\langle\left[j_{\mu}(x), j_{\nu}\left(x^{\prime}\right)\right]\right\rangle_{0} \epsilon\left(x-x^{\prime}\right)\right\}=0 .
$$

Schwinger tried to prove the validity of this condition, after reducing it to the form

$$
\left\langle\left[j_{\mu}(x), j_{\nu}\left(x^{\prime}\right)\right]\right\rangle_{0}\left[\partial \epsilon\left(x-x^{\prime}\right) / \partial x_{\mu}\right]=0,
$$

${ }^{1}$ S. Tomonaga, Prog. Theor. Phys. 1, 27 (1946). J. Schwinger, Phys. Rev. 74, 1439 (1948); Phys. Rev. 75, 651 (1949); Phys. Rev. 75, 1912 (1949). These papers are quoted in the following as SI, SII, SIII. Our notations follow as closely as possible those of these papers. For the definitions and the properties of the functions $\Delta, \boldsymbol{\Delta}:(\boldsymbol{\Delta}$ is identical with $\bar{\Delta}$ in SII $)$, and $\Delta^{(1)}$ see particularly the appendix of SII. In this paper natural units $\hbar=c=1$ are used throughout. F. J. Dyson, Phys. Rev. 75, 486 (1949), and Phys. Rev. 75, 1736 (1949). In the following quoted as DI, and DII.

${ }^{2}$ G. Wentzel, Phys. Rev. 74, 1070 (1948).

${ }^{3}$ SII, Eq. (2.19). by the argument that a time-like component of the current commutes with $j_{\nu}$ at all points of a space-like surface. ${ }^{4}$ The specialization of the general invariant form of the commutator to this case, however, gives a result proportional to

$$
\delta^{(3)}\left(x-x^{\prime}\right)\left(\partial \Delta^{(1)} / \partial x_{\nu}\right),
$$

which is indeterminate due to the singularity of $\partial \Delta^{(1)} / \partial x_{\nu}$ on the light cone, which has the form $\sim x_{\nu} /\left(x_{\sigma} x_{\sigma}\right)$. The whole expression may therefore be written as

$$
\delta^{(4)}\left(x-x^{\prime}\right)\left(\partial \Delta^{(1)} / \partial x_{v}\right)
$$

in agreement with the straightforward computation (see $\$ 2$ below).

The occurrence of products of functions with a $\delta$-type singularity and with a pole is typical of the new formalism and seems to be the main source of the remaining uniqueness problems.

In order to overcome these ambiguities we apply in the following the method of regularization of $\Delta$-functions (or products of them) with the help of an introduction of auxiliary masses. This method has already a long history. Much work has been done to compensate the infinities in the self-energy of the electron with the help of auxiliary fields corresponding to other neutral particles with finite rest-masses interacting with the electrons. ${ }^{5}$ Some authors assumed formally a negative energy of the free auxiliary particles, while others did not need these artificial assumptions and could obtain the necessary compensations by using the different sign of the self-energy of the electron due to its interaction with different kinds of fields (for instance scalar fields $v s$. vector fields). We shall denote these theories, in which the auxiliary particles with finite masses and positive energy are assumed to be observable in principle and are described by observables entering the Hamil-

${ }^{4}$ SII, Eq. (2.29)

5 Compare for older literature (including his own contributions), A. Pais, The Development of the Theory of the Electron (Princeton University Press, Princeton, New Jersey, 1948). 
tonian explicitly as "realistic," in contrast to "formalistic" theories, in which the auxiliary masses are used merely as mathematical parameters which may finally tend to infinity. Recently the "realistic" standpoint was extended to the problem of the cancellation of the singularities in the vacuum polarization, due to virtual electron-positron pairs generated by external fields, by introducing auxiliary pairs of particles with opposite electric charges and masses different from that of the electron. ${ }^{6}$ It was shown that the signs of the polarization effect allow compensation of the singularities only if the auxiliary particles are assumed to obey Bose-Statistics. Until now it was not possible to carry through the "realistic" standpoint to include all possible effects in higher order approximations in the fine-structure constant, nor is it proven that this problem is not overdetermined. Presumably a consistent "realistic" theory will only be possible if, from the very beginning, all observables entering the theory have commutation rules and vacuum expectation values free from singularities, i.e., different from the $\boldsymbol{\Delta}$ and $\Delta^{(1)}$ functions which obey a wave equation corresponding to a given mass value. Until now, however, it has not been possible to carry through such a program.

At the present stage of our knowledge it is therefore of interest to investigate further the "formalistic" use of auxiliary masses in relativistic quantum theory. This was done independently by Feynman ${ }^{7}$ and by Stueckelberg and Rivier. ${ }^{8}$ The latter authors use (more generally) an arbitrary number of auxiliary masses, while the former introduces only a single large auxiliary mass, which was sufficient for his particular problem, the regularization of the self-energy of the electron. From the well-known expansions of the $\Delta$ - and $\Delta^{(1)}$ functions near the light cone it can easily be seen (see $\$ 2)$ that in the linear combinations:

and

$$
\boldsymbol{\Delta}_{R}(x)=\sum_{i} c_{i} \mathbf{\Delta}\left(x ; M_{i}\right)
$$

$$
\Delta_{R}^{(1)}(x)=\sum_{i} c_{i} \Delta^{(1)}\left(x ; M_{i}\right)
$$

the strongest singularities cancel, if

$$
\sum_{i} c_{i}=0 \text {, }
$$

and the remaining singularities (finite jumps and logarithmic singularities) also cancel if in addition the condition

$$
\sum_{i} c_{i} M_{i}^{2}=0
$$

holds. If the first condition alone is sufficient to guarantee the regularity of a certain result, it is obvious that a single auxiliary mass $M_{1}$ (besides the original electron

${ }^{6}$ G. Rayski, Acta Phys. Polonica 9, 129 (1948). (Only light waves as external electromagnetic field are considered in this paper.) Umesawa, Yukawa, and Yamada, Prog. Theor. Phys. 3, No. 3, 317 (1948).

7 R. P. Feynman, Pocono Conference 1948; Phys. Rev. 74, 1439 (1948). Applications by V. F. Weisskopf and J. B. French, Phys. Rev. 75, 1240 (1949).

8 E. C. G. Stueckelberg and D. Rivier, Phys. Rev. 74, 218 and 986 (1948). D. Rivier, Helv. Phys. Acta XXII, 265 (1949). mass $M_{0}=m$ ) with $c_{1}=-c_{0}=-1$ is all that is necessary. It should not be forgotten, however, that Feynman's success in using a single auxiliary mass in the problem of the self-energy of the electron implies the assumption that in the corresponding expression ${ }^{9}$ resulting from the invariant form of perturbation theory the photon functions $\bar{D}$ and $D^{(1)}$ have both to be regularized with the same auxiliary mass. (A formal alternative would be to leave the photon-functions unchanged, but to regularize the electron-functions $\Delta$ and $\Delta^{(1)}$ with the same auxiliary mass, or to regularize the whole expression without factorization and with one auxiliary mass.)

The application of the formal method of massregularization to the problem of vacuum-polarization ${ }^{10}$ (\$4) shows that not only the use of a single auxiliary mass is here insufficient, but that any regularization of $\Delta$ - or $\Delta^{(1)}$-functions as separate factors leads to results that are not gauge invariant. As was shown by Rayski ${ }^{11}$ only the regularization of the whole expression for the resulting current (without factorization) gives satisfactory results in this case. The formal use of continuous mass distributions is here particularly suited to illustrate the connection between the different results of Wentzel and Schwinger for the photon self-energy.

In $\$ 5$ the example of the correction to the magnetic moment of the electron, which is one of the main results of Schwinger, is treated from the "formalistic" standpoint of mass regularization. We agree with Schwinger that the use of auxiliary masses is not necessary in this case if the computations in momentum space are made with sufficient care (see additional remark A). In any case (different from the situation in the problem of vacuum-polarization), the use of a single auxiliary mass is here sufficient to avoid any ambiguity, provided that the same mass is applied both to the $\bar{D}$ and the $D^{(1)}$-functions of the photon, analogous to Feynman's method for the self-energy of the electron, or that the regularization is applied to the products of two $\Delta$ - and $\Delta^{(1)}$-functions without factorization $^{12}$ (see reference 20 ).

${ }^{9}$ SII, Eqs. (3.77) and (3.82).

${ }^{10}$ Dyson (see DII) applies to this problem a method of regularization without use of auxiliary masses, which is more similar to the methods used in the earlier stages of positron theory.

11 Rayski made this proposal in the summer of 1948, during his investigations on the photon self-energy of Bosons (see reference 6 ). With his friendly consent we later resumed his work and generalized the method for arbitrary external fields (not necessarily light waves).

${ }_{12}$ The problem of the magnetic moment of nucleons due to a mesonic interaction, which shows a close analogy to the problem of the magnetic moment of the electron due to electromagnetic interaction, is not treated in this paper. Stueckelberg-Rivier give (see reference 8) a formula for the magnetic moment of the neutron which they characterize as not leading to a definite numerical value. A justification of this may, in principle, be seen in the fact that the most general form of regularization with auxiliary masses must always lead to an arbitrary value for integrals of this type. On the other hand the mentioned general analogy between the two cases makes it plausible that the same mathematical methods which lead to an unambiguous definition of the magnetic moment of the electron will also lead to an unique definition of the value of the theoretical results for the magnetic moments of the nucleons (at least for scalar and pseudo- 
Both groups of authors (Stueckelberg-Rivier ${ }^{8}$ and Feynman-Dyson ${ }^{13}$ ) seem to ascribe to a particular combination of $\Delta$-functions, which describes outgoing waves for the future and incoming waves for the past, an important or even fundamental significance. As this question can be left open for the purpose of this paper, we discuss Dyson's expression for the magnetic moment of the electron, in which the function $\Delta_{c}$ for the electron and $D_{c}$ for the photon alone occur,,$^{14}$ only in a brief additional remark $(\mathrm{B} ; \S 5)$. We believe that in order to investigate the range of applicability of the particular function $\Delta_{c}$, the discussion of more complicated examples will be necessary.

Summarizing, one must admit that the additional rules which the "formalistic" standpoint has to use (e.g., to apply the same mass values for $\boldsymbol{\Delta}$ - and $\Delta^{(1)}$ functions, and not to factorize the regularization of products of $\Delta$ - and $\Delta^{(1)}$-functions corresponding to pairs of charged particles) could be immediately understood from the "realistic" standpoint and appears as if borrowed from the latter. ${ }^{15}$ It seems very likely that the "formalistic" standpoint used in this paper and by other workers can only be a transitional stage of the theory, and that the auxiliary masses will eventually either be entirely eliminated, or the "realistic" standpoint will be so much improved that the theory will not contain any further accidental compensations.

\section{§2. THE BASIC CONCEPTS OF REGULARIZATION}

In an invariant perturbation theory, such as the one introduced by Schwinger into quantum electrodynamics, the two invariant functions, $\Delta$ and $\Delta^{(1)}$, play an essential role. Vacuum expectation values of properly symmetrized products of field operators are expressed in terms of $\Delta^{(1)}$, while $\boldsymbol{\Delta}$ appears in connection with the covariant formulation of commutation rules.

The handling of expressions involving $\Delta$ - and $\Delta^{(1)}$

scalar mesons). Meanwhile K. M. Case, Phys. Rev. 76, 1 (1949), obtains an unambiguous result (from invariant perturbation theory) for the magnetic nucleon moments which agrees completely with those of Luttinger (Helv. Phys. Acta XXI, 483 (1948)). He does not give the details of his evaluation of the integrals, for which no auxiliary masses are needed.

${ }^{13}$ Compare DI and DII. The function in question is denoted with $D_{c}$ by Stueckelberg-Rivier, with $D_{F}$ by Dyson and with $\Delta_{F}$ by Case. We use the notation $\Delta_{c}$ and $D_{c}$ for the corresponding electron and photon functions respectively.

${ }_{14} \mathrm{DI}$, Section X, formula for $L$.

15 The interesting problem of the "self-stress" of the electron (see A. Pais, reference 5; in an unpublished letter of last year Pais gave the result that in the theory of holes the value of this self-stress is finite, namely $\alpha / 2 \pi \cdot m$ ( $\alpha=$ fine-structure constant), but not zero, as special relativity requires for the total stress of a closed system) may throw more light on the relations between the two standpoints. Detailed calculations by one of us (F.V.) gave the result that a formal regularization with auxiliary masses does not change the finite value of Pais for the self-stress; one has therefore either to consider the localization of energy in space and time as a non-physical concept in quantum theory and to admit only the energy-momentum vector (which is already integrated over space-time), or one has to ascribe to the compensating auxiliary masses a physical reality such that their contribution to the stress in the intermediate states compensates the other part of the self-stress of the electron. functions exhibit some characteristic difficulties, which may be summarized as follows:

(a) The occurrence of indeterminate expressions as a consequence of the coincidence of the $\delta$-type singularity of $\boldsymbol{\Delta}(x)$ with the pole of $\Delta^{(1)}(x)$ on the light cone. Only a properly defined limiting process may give them a definite meaning.

(b) The necessity of taking into account, in the course of the calculation, the "covariance" of some diverging (however formally covariant) expression in order to split off a finite part. This too may be done in a proper way only after these expressions have been made finite by a regularization process.

Since both difficulties are connected with the singular features of the $\boldsymbol{\Delta}$ - and $\Delta^{(1)}$-functions on the light cone, an invariant elimination of these singularities may be helpful in an attempt to escape the above-mentioned complications. Looking for such a device, one is guided by the dependence of $\boldsymbol{\Delta}$ and $\Delta^{(1)}$ on the rest mass of the corresponding field. This dependence is exhibited in the integral representations:

$$
\begin{gathered}
\Delta^{(1)}(x)=-\left(m^{2} / 2 \pi^{2}\right) \int_{0}^{\infty} d \alpha \sin \left[\lambda m^{2} \cdot \alpha+(1 / 4 \alpha)\right] \\
\Delta(x)=\left(m^{2} / 4 \pi^{2}\right) \int_{0}^{\infty} d \alpha \cos \left[\lambda m^{2} \cdot \alpha+(1 / 4 \alpha)\right]
\end{gathered}
$$

where

$$
\lambda=-x_{\mu} x_{\mu}, *
$$

which show that both $\Delta^{(1)}$ and $\boldsymbol{\Delta}$ are of the form:

$$
m^{2} \cdot f u\left(\lambda m^{2}\right) \text {. }
$$

From this it follows that $\delta$-type singularities $(\delta(\lambda))$ and first order poles $(1 / \lambda)$ are independent of $m$, whereas finite jumps and logarithmic singularities are proportional to $\mathrm{m}^{2}$. Since these are the only types of singularities occurring in $\boldsymbol{\Delta}$ and $\Delta^{(1)}$, they may be avoided by introducing the regularized invariant functions $\Delta_{R}$ and $\Delta_{R}{ }^{(1)}$ is:

$$
\Delta_{R}^{(1)}=\sum_{i} c_{i} \Delta^{(1)}\left(M_{i}\right), \quad \Delta_{R}=\sum_{i} c_{i} \Delta\left(M_{i}\right),
$$

where $c_{i}$ satisfies the conditions:

$$
\begin{aligned}
\sum_{i} c_{i} & =0 \\
\sum_{i} c_{i} M_{i}{ }^{2} & =0 .
\end{aligned}
$$

In order to exhibit more clearly the efficacy of these conditions we give the development of $\Delta^{(1)}$ and $\boldsymbol{\Delta}$ for small $\lambda$ (omitting all terms vanishing for $\lambda=0$ ):

$$
\begin{aligned}
\Delta^{(1)}(x) & =\frac{1}{4 \pi}\left\{\frac{-2}{\lambda}+m^{2} \log \frac{\gamma}{2}\left(|\lambda| m^{2}\right)^{\frac{1}{2}}-\frac{m^{2}}{2}+\cdots\right\} \\
\Delta(x) & =\frac{1}{4 \pi}\left\{\delta(\lambda)+\left(\frac{m^{2}}{4}+\cdots\right) \theta^{+}(\lambda)\right\}
\end{aligned}
$$

where

$$
\theta^{+}(x)=\left\{\begin{array}{l}
1 \\
0
\end{array}\right\} \quad \text { for } \quad x \gtrless 0 .
$$

* In the following $x_{4}=i x_{0}=i t$. 
It is easily seen that $\Delta_{R}$ vanishes for $\lambda=0$, such that

$$
\Delta(x)=-2 \epsilon(x) \Delta(x)
$$

is regularized too. $\Delta_{R}^{(1)}$, however, takes the value

$$
\frac{1}{4 \pi} \sum_{i} c_{i} M_{i}^{2} \log M_{i} \text { for } \lambda=0 .
$$

It is the meaning of the regularization prescriptions that the first term in the series (3) represents the non-regularized function itself, i.e., that

$$
c_{0}=1, \quad M_{0}=m
$$

and that all $M_{i}(i>0)$ should finally tend to $\infty$ (according to the "formalistic" standpoint, adopted in the following). The coefficients $c_{i}$ need hereby not remain finite. We shall, however, impose the condition

$$
\sum_{i}^{\prime}\left(\left|c_{i}\right| / M_{i}^{2}\right) \rightarrow 0
$$

which ensures that

$$
\begin{array}{ll}
\sum_{i}{ }^{\prime} c_{i} F\left(M_{i}{ }^{2}\right) \rightarrow 0 & \text { if only } \\
\left|M_{i}{ }^{2} F\left(M_{i}^{2}\right)\right|<A & \text { for all } i>0 .
\end{array}
$$

For the purposes of a general discussion it may sometimes be advantageous to replace the discrete spectrum of auxiliary masses by a continuous one (including, or course, the discrete as a special case):

$$
\boldsymbol{\Delta}_{R}(x)=\int_{-\infty}^{+\infty} d \kappa \rho(\kappa) \Delta(x ; \kappa), \text { etc. }
$$

where $\kappa$ has the signification of the square of a mass.

The conditions (I, Ia) now read:

$$
\begin{aligned}
& \int d \kappa \rho(\kappa)=0 \\
& \int d \kappa \kappa \rho(\kappa)=0 .
\end{aligned}
$$

On writing $\rho(\kappa)$ as $\delta\left(\kappa-m^{2}\right)+\rho_{1}(\kappa)$ condition (6) takes the form

$$
\int_{a}^{\infty} d \kappa\left(\left|\rho_{1}(\kappa)\right| / \kappa\right) \rightarrow 0 . \quad a>0
$$

An alternative possibility of regularization is contained in the prescription

$$
F_{R}=\int d \kappa \rho(\kappa) F\left(\Delta^{(1)}(\kappa), \Delta(\kappa)\right)
$$

where $F$ represents some (bilinear or higher order) form in $\boldsymbol{\Delta}, \Delta^{(1)}, d \mathbf{\Delta} / d \lambda, d \Delta^{(1)} / d \lambda$.

The use of the prescription (9) needs further explanation: The form $F$ may contain both $\Delta$-(electron) and $D$-(photon) functions. We may then either regularize the expression $F$ as a whole (both $\Delta$ - and $D$-functions without factorization):

$$
\begin{aligned}
& F_{R}=\int d \kappa \rho(\kappa) F\left(\Delta^{(1)}\left(\kappa^{\prime}\right), D^{(1)}(\kappa) ; \Delta\left(\kappa^{\prime}\right), \bar{D}(\kappa)\right) \\
& \kappa^{\prime}=\left(m^{2}+\kappa\right)^{\frac{1}{2}},
\end{aligned}
$$

or we may also regularize $F$ only with respect to the $D$-function referring to one type of field, e.g.:

$$
F_{R}=\int d \kappa \rho(\kappa) F\left(\Delta^{(1)}(m), D^{(1)}(\kappa) ; \Delta(m), \bar{D}(\kappa)\right) .
$$

Whenever in this latter case $F$ is linear with respect to the $D$-function to be regularized, (9) reduces to (3) (i.e., the introduction of individually regularized $D$ functions), but implies the important additional rule that the same regulator $\rho(\kappa)$ has to be applied to both $\bar{D}$ and $D^{(1)}$. If, on the contrary, $F$ is bilinear with respect to the field in question, all these bilinear terms have to be regularized without factorization and with the same regulator. In this latter case the conditions $\left(\mathrm{I}^{\prime}\right),\left(\mathrm{I}^{\prime} \mathrm{a}\right)$ are then, in general, not sufficient to remove all singularities from (9). They eliminate however the strongest ones, especially those of the type $\delta(\lambda) / \lambda$.

The rule (9), interpreted in the above-explained sense, will be adopted in the following throughout. It is this rule that assures the gauge invariance of the polarization current in the problem of vacuum polarizationin contrast to the results of (3).

One may object that this prescription suffers from a lack of uniqueness, but this apparent deficiency affects only the mass and charge renormalization terms. Hereby we mean, more precisely, that after mass and charge terms have been removed, all additional corrective terms shall be independent of the way they are regularized, and shall, of course, be independent of the parameters $c_{i}$ and $M_{i}$ in the limit $M_{i} \rightarrow \infty$ (or $\rho_{1}(\kappa) \rightarrow 0$ for any finite $\left.\kappa\right)$. This is not the case if in the form $F,(9)$, the individual summands are regularized independently and differently:

$$
F=F_{1}+F_{2} ; \quad F_{R}=\int d \kappa \rho_{a}(\kappa) F_{1}(\kappa)+\int d \kappa \rho_{b}(\kappa) F_{2}(\kappa) ;
$$

a quite arbitrary result may then be obtained, as will be shown later on (see $\$ 5$, additional remark $\mathrm{C}$ ). The charge and mass terms themselves, however, depend on the way they are regularized, since they depend on

$$
\sum_{i} c_{i} \log M_{i} \text { or } \int d \kappa \rho(\kappa) \log \kappa
$$

In connection with the use of the Fourier-integral representation of $\boldsymbol{\Delta}$ and $\Delta^{(1)}$ (as in $(1 \mathrm{a}, \mathrm{b})$ ) for computational purposes, it is convenient to have conditions $\left(I^{\prime}, I^{\prime} a\right)$ expressed in terms of the Fourier transformed of $\rho(\kappa)$ :

$$
R(a)=\int d \kappa \rho(\kappa) e^{-i a \kappa}
$$


The conditions $\left(I^{\prime}, I^{\prime} a\right)$ then read

$$
\begin{aligned}
R(0) & =0 \\
R^{\prime}(0) & =0
\end{aligned}
$$

while the integral (10) is transformed into

$$
\begin{aligned}
& -\frac{1}{2} \int_{-\infty}^{+\infty} \frac{d a}{a} \epsilon(a) R(a) . \\
& (\epsilon(a)= \pm 1 \text { for } a \gtrless 0) .
\end{aligned}
$$

\section{\$3. THE INVARIANT PERTURBATION THEORY}

Let $\psi(x), \bar{\psi}(x)=\psi^{*}(x) \beta$ be the quantized operators of the electron field and $A_{\mu}(x)$ the four potential representing the radiation field. $\psi, \bar{\psi}$ and $A_{\mu}$ are supposed to satisfy the equations of the uncoupled fields:

$$
\begin{aligned}
{\left[\gamma_{\nu}\left(\partial / \partial x_{\nu}\right)+m\right] \psi } & =0, \\
\left(\partial \bar{\psi} / \partial x_{\nu}\right) \gamma^{\nu}-m \bar{\psi} & =0, \\
\square A_{\mu} & =0, \\
\left(\partial A_{\mu} / \partial x_{\mu}\right) \Psi(t) & =0,
\end{aligned}
$$

and accordingly, obey the commutation relations:

$$
\begin{aligned}
{\left[A_{\mu}(x), A_{\nu}\left(x^{\prime}\right)\right] } & =i \delta_{\mu \nu} D\left(x-x^{\prime}\right) \\
\left\{\psi_{\alpha}(x), \bar{\psi}_{\beta}\left(x^{\prime}\right)\right\} & =\frac{1}{i} S_{\alpha \beta}\left(x-x^{\prime}\right) . \\
& =\frac{1}{i}\left(\gamma^{\nu} \frac{\partial}{\partial x_{\nu}}-m\right)_{\alpha \beta} \Delta\left(x-x^{\prime}\right) . \\
{[A, B] } & =A B-B A, \quad\{A, B\}=A B+B A .
\end{aligned}
$$

The auxiliary condition (14) involves the state vector $\Psi$ of the system, whose equation of motion is given by

$$
i(\partial \Psi / \partial t)=H \Psi .
$$

Herein $H$ represents the interaction energy:

$$
\begin{aligned}
H & =-\int d^{3} x j_{\mu}(x) A_{\mu}(x) \\
& =-i e \int d^{3} x \bar{\psi}(x) \gamma^{\mu} \psi(x) \cdot A_{\mu}(x) .
\end{aligned}
$$

The solution of (17) may be achieved in successive approximations by a set of unitary transformations:

$$
\Psi=e^{-i S_{1}(t)} \Psi_{1}=e^{-i S_{1}(t)} e^{-i S_{2}(t)} \Psi_{2}=\cdots=U(t) \Psi_{K},
$$

where $\Psi_{K}$ is time-independent in the desired approximation and thus represents some "free-particle"-state.

The change in properties of an operator $\Omega$ referring say to the field $a$, due to the interaction of $a$ with the vacuum of the field $b$, is then expressed in the vacuum expectation value (with respect to $b$ ) of the transformed operator $\mathbf{\Omega}$ :

$$
\langle\boldsymbol{\Omega}\rangle_{\operatorname{vac}(b)}=\left\langle U^{-1} \Omega u\right\rangle_{\operatorname{vac}(b)} .
$$

Sometimes it may be convenient to separate the electromagnetic field (and its sources) from the system under consideration and treat it as a given ( $c$-number) field: $A_{\mu}{ }^{\operatorname{ext}}(x)$, satisfying:

$$
\square{ }^{2} A_{\mu}{ }^{\mathrm{ext}}(x)=-J_{\mu}^{\mathrm{ext}}(x) .
$$

As before, "states" are represented by a time-independent state vector $\Psi_{K}$ and a transformation formally analogous to (19), introducing $\Psi_{K}$, exhibits the change, induced by the presence of $A_{\mu}{ }^{\mathrm{ext}}$, in the expectation value of an operator $\Omega$.

The transformation $u$, (19), shall be written, more precisely, as

$$
u(t)=e^{-i S_{1}(t)} N_{1} e^{-i S_{2}(t)} N_{2} \cdots,
$$

where $S_{1}(t)$ is thought to remove the first-order coupling, $S_{2}(t)$ the remaining second-order interaction, etc. Between these steps may take place renormalization transformations $N$, defining new matter field operators which obey equations with adjusted mass.

According to this program, $S_{1}$ is defined by:

$$
\dot{S}_{1}=H
$$

leaving for $\Psi_{1}$ the equation of motion

$i\left(\partial \Psi_{1} / \partial t\right)=\left(i / 2\left[S_{1}, H\right]+\cdots\right) \Psi_{1}$.

Then

$$
\dot{S}_{2}=i / 2\left[S_{1}, H\right]-H_{\text {self }}
$$

leading thus to a state vector $\Psi_{2}$ which is constant in time up to and including terms in $e^{2}$, provided a mass renormalization has taken place, removing $H_{\text {self }}$ from $i / 2\left[S_{1}, H\right]$.

Restricting ourselves to this order of approximation, a transformed operator $\boldsymbol{\Omega}$, according to (20), may be written as follows:

$$
\boldsymbol{\Omega}=\Omega+i\left[S_{1}, \Omega\right]-\frac{1}{2}\left[S_{1}\left[S_{1}, \Omega\right]\right]+i\left[S_{2}, \Omega\right]
$$

$$
\begin{aligned}
\boldsymbol{\Omega}(t)= & \Omega(t)+i \int^{t} d t^{\prime}\left[H\left(t^{\prime}\right), \Omega(t)\right] \\
& -\frac{1}{2} \int^{t} d t^{\prime} \int^{t} d t^{\prime \prime}\left[H\left(t^{\prime}\right)\left[H\left(t^{\prime \prime}\right), \Omega(t)\right]\right] \\
& +i \int^{t} d t^{\prime}\left[-\frac{i}{2}\left[S_{1}\left(t^{\prime}\right), H\left(t^{\prime}\right)\right]-H_{s}\left(t^{\prime}\right), \Omega(t)\right]
\end{aligned}
$$

$$
\begin{aligned}
\text { or } \\
\mathbf{\Omega}(t)=\Omega(t)+i \int^{t} d t^{\prime}\left[H\left(t^{\prime}\right), \Omega(t)\right] \\
-\int^{t} d t^{\prime} \int^{t^{\prime}} d t^{\prime \prime}\left[H\left(t^{\prime \prime}\right)\left[H\left(t^{\prime}\right), \Omega(t)\right]\right] \\
-i \int^{t} d t^{\prime}\left[H_{s}\left(t^{\prime}\right), \Omega(t)\right] .
\end{aligned}
$$

** To obtain this latter form a simplification due to Schwinger has been used, which employs Jacobi's identity. 
With the help of the commutators (15), (16) and the derived relation:

$$
\begin{aligned}
& {\left[\bar{\psi}(x) \gamma^{\mu} \psi(x), \bar{\psi}\left(x^{\prime}\right) \gamma^{\nu} \psi\left(x^{\prime}\right)\right]} \\
& =1 / i\left\{\bar{\psi}(x) \gamma^{\mu} S\left(x-x^{\prime}\right) \gamma^{\nu} \psi\left(x^{\prime}\right)\right. \\
& \left.-\bar{\psi}\left(x^{\prime}\right) \gamma^{\nu} S\left(x^{\prime}-x\right) \gamma^{\mu} \psi(x)\right\},
\end{aligned}
$$

the transformed operator $\boldsymbol{\Omega}$ is easily evaluated. To have its expectation value for a definite state (for instance the vacuum with respect to one of the fields in question) we need still know the vacuum expectation values of the properly symmetrized products:

$$
\begin{aligned}
\left\langle\left\{A_{\mu}(x), A_{\nu}\left(x^{\prime}\right)\right\}\right\rangle_{0} & =\delta_{\mu \nu} D^{(1)}\left(x-x^{\prime}\right) \\
\left\langle\left[\psi_{\alpha}(x), \bar{\psi}_{\beta}\left(x^{\prime}\right)\right]\right\rangle_{0} & =-S_{\alpha \beta}{ }^{(1)}\left(x-x^{\prime}\right) .
\end{aligned}
$$

As examples which will be discussed later on, we give:

(a) The current induced in the (matter-) vacuum by an external electromagnetic field $A_{\mu}{ }^{\text {ext }}$, or its source $J_{\mu}{ }^{\text {ext }}$. The desired approximation is achieved with $S_{1}$ and yields immediately:

$$
\begin{aligned}
\left\langle j_{\mu}(x)\right\rangle=i \int^{t} d t^{\prime}\langle & {\left.\left[H^{\operatorname{ext}}\left(t^{\prime}\right), j_{\mu}(x)\right]\right\rangle_{0} } \\
& =-4 e^{2} \int d^{4} x^{\prime} K_{\mu \nu}\left(x-x^{\prime}\right) A_{\nu}^{\text {ext }}\left(x^{\prime}\right)
\end{aligned}
$$

where

$$
K_{\mu \nu}\left(x-x^{\prime}\right)=\frac{1}{8 i e^{2}}\left\langle\left[j_{\mu}(x), j_{\nu}\left(x^{\prime}\right)\right]\right\rangle_{0} \epsilon\left(x-x^{\prime}\right) .
$$

(b) The $e^{2}$-radiative correction to the expression for the current associated with the matter field. According to (25) this correction is contained in

$$
\begin{aligned}
\Delta j_{\mu}(x)=-\int^{t} d t^{\prime} \int^{t^{\prime}} d t^{\prime \prime} & \left\langle\left[H\left(t^{\prime \prime}\right)\left[H\left(t^{\prime}\right), j_{\mu}(x)\right]\right]\right\rangle_{\text {phot vac }} \\
& -i \int^{t} d t^{\prime}\left[H_{\text {self }}\left(t^{\prime}\right), j_{\mu}(x)\right] .
\end{aligned}
$$

The one particle part (for the definition of the concept of the one particle part of an operator, see SII, page 671) of $\Delta j_{\mu}(x)$ includes a term of the form

$$
j_{\mu}^{(s)}(x)=\text { const. } \frac{\partial}{\partial x_{\nu}}\left(\bar{\psi}(x) \sigma^{\mu \nu} \psi(x)\right),
$$

describing an anomalous $g$-factor of the electron.

\section{\$4. VACUUM POLARIZATION AND PHOTON SELF-ENERGY}

In this section the tensor $K_{\mu \nu}\left(x-x^{\prime}\right)(30)$ shall be investigated. Invariance of the induced current with

*** The factor $\epsilon\left(x-x^{\prime}\right)$ is introduced by writing:

$$
\int_{-\infty}^{t} d t^{\prime}=\frac{1}{2} \int_{-\infty}^{+\infty} d t^{\prime} \epsilon\left(t-t^{\prime}\right)+\frac{1}{2} \int_{-\infty}^{+\infty} d t^{\prime} ;
$$

the second integral vanishes, if no real transition is induced by the external field. respect to the gauge transformation $A_{\mu} \rightarrow A_{\mu}+\left(\partial \Lambda / \partial x_{\mu}\right)$ gives

$$
0=\int d^{4} x^{\prime} K_{\mu \nu}\left(x-x^{\prime}\right)\left(\partial \Lambda / \partial x_{\nu}{ }^{\prime}\right),
$$

which requires:

$$
\frac{\partial K_{\mu \nu}(x)}{\partial x_{\nu}}=0 .
$$

In momentum space, where (29) reads:

$$
\left\langle j_{\mu}(p)\right\rangle=-4 e^{2} K_{\mu \nu}(p) A_{\nu}(p),
$$

the condition (33) is equivalent to

On writing:

$$
K_{\mu \nu}(p) \cdot p_{\nu}=0
$$

$$
K_{\mu \nu}(p)=K_{1} p_{\mu} p_{\nu}+K_{2} \delta_{\mu \nu} p_{\lambda} p_{\lambda} .
$$

Equation $\left(33^{\prime}\right)$ is equivalent to the condition

$$
K_{1}=-K_{2}
$$

(both $K_{1}$ and $K_{2}$ are still functions of the invariant $p_{\lambda} p_{\lambda}$ ). Since $A_{\mu}{ }^{\text {ext }}$ and the current $J_{\mu}{ }^{\text {ext }}$ generating this external field are connected through

$$
J_{\mu}{ }^{\text {ext }}(p)=\left(\delta_{\mu \nu} p_{\lambda} p_{\lambda}-p_{\mu} p_{\nu}\right) A_{\mu}{ }^{\text {ext }}(p),
$$

condition (36) assures that:

$$
\left\langle j_{\mu}(p)\right\rangle=4 e^{2} K_{1} J_{\mu}{ }^{\operatorname{ext}}(p) .
$$

An evaluation of $K_{\mu \nu}$ with the help of relations (26) and (28) gives:

$$
\begin{aligned}
K_{\mu \nu}(x)=\frac{\partial \Delta}{\partial x_{\mu}} \cdot \frac{\partial \Delta^{(1)}}{\partial x_{\nu}}+ & \frac{\partial \Delta}{\partial x_{\nu}} \cdot \frac{\partial \Delta^{(1)}}{\partial x_{\mu}} \\
& -\delta_{\mu \nu}\left(\frac{\partial \Delta}{\partial x_{\lambda}} \cdot \frac{\partial \Delta^{(1)}}{\partial x_{\lambda}}+m^{2} \Delta^{(1)} \Delta\right) .
\end{aligned}
$$

From the expansions $(4,5)$ it follows that this expression is indeterminate on the light cone, due to terms of the type $\delta(\lambda) / \lambda$, and the relation (33) yields, on account of

$$
\begin{gathered}
\left(\square^{2}-m^{2}\right) \Delta^{(1)}=0, \quad\left(\square^{2}-m^{2}\right) \Delta=-\delta(x) ; \\
\frac{\partial K_{\mu \nu}(x)}{\partial x_{\nu}}=-\frac{\partial \Delta^{(1)}}{\partial x_{\mu}} \delta(x),
\end{gathered}
$$

which is indeterminate, too.

In momentum space, where $K_{\mu \nu}$ is given by

$$
\begin{aligned}
K_{\mu \nu}(p)=\frac{1}{(2 \pi)^{3}} \int & d^{4} k \frac{\delta\left(k_{\lambda} k_{\lambda}+m^{2}\right)}{\left(k_{\lambda}-p_{\lambda}\right)^{2}+m^{2}}\left\{2 k_{\mu} k_{\nu}-k_{\mu} p_{\nu}\right. \\
& \left.-k_{\nu} p_{\nu}-\delta_{\mu \nu}\left(-k_{\lambda} p_{\lambda}+k_{\lambda} k_{\lambda}+m^{2}\right)\right\}
\end{aligned}
$$

this ambiguity is less manifest, since

$$
K_{\mu \nu}(p) \cdot p_{\nu}=\frac{1}{(2 \pi)^{3}} \int d^{4} k k_{\mu} \delta\left(k_{\lambda} k_{\lambda}+m^{2}\right)
$$


(hereby terms of the form $\delta\left(k_{\lambda} k_{\lambda}+m^{2}\right) \cdot\left(k_{\lambda} k_{\lambda}+m^{2}\right)$ have been omitted), an expression which may well be put equal to zero for symmetry reasons. Since, however, $K_{\mu \nu}(\rho)$ is represented by a divergent Fourier-integral, this property may be lost in the course of a direct computation of $K_{\mu \nu}$. Note that conditions (I,I a) are just those necessary to make the integral (39) convergent!

An evaluation of $K_{\mu \nu}$ is most conveniently done with the help of the Fourier-integral representations: $:^{16}$

$\Delta^{(1)}(k)=2 \pi \delta\left(k_{\lambda} k_{\lambda}+m^{2}\right)$

$$
=\int_{-\infty}^{+\infty} d \alpha \exp \left[i \alpha\left(k_{\lambda} k_{\lambda}+m^{2}\right)\right]
$$

$$
\begin{aligned}
& \Delta(k)=P \frac{1}{k_{\lambda} k_{\lambda}+m^{2}} \\
& \quad=\frac{1}{2 i} \int_{-\infty}^{+\infty} d \beta \epsilon(\beta) \exp \left[i \beta\left(k_{\lambda} k_{\lambda}+m^{2}\right)\right]
\end{aligned}
$$

( $P=$ principal value). After introducing a new variable

$$
k^{\prime}=k-(\beta / \alpha+\beta) \cdot p
$$

and with

$$
\begin{gathered}
\int d^{4} k \exp \left(i a k_{\lambda} k_{\lambda}\right)=\left(i \pi^{2} / a^{2}\right) \epsilon(a) \\
\int d^{4} k k_{\mu} k_{\nu} \exp \left(i a k_{\lambda} k_{\lambda}\right)=-\delta_{\mu \nu}\left(\pi^{2} / 2 a^{3}\right) \epsilon(a),
\end{gathered}
$$

we are left (after symmetrizing the integrand with

${ }^{16}$ S. T. Ma (Phys. Rev. 75, 1264 (1949)) has evaluated $K_{\mu \nu}(p)$ by means of elementary momentum-space integrations, using the method due to Pauli and Rose (Phys. Rev. 49, 462 (1936)). His results are neither gauge nor Lorentz-invariant, due to the presence of an additional constant term $\boldsymbol{\Gamma}$ in his expression for $K_{i i}(i=1,2,3)$. As a consequence, this term appears also in the trace of $K_{\mu \nu}$ :

$$
K_{\mu \mu}=-3 p_{\mu} p_{\mu} K_{1}+3 \Gamma,
$$

whereas, according to (35) and (36) $K_{\mu \mu}$ should be proportional to $\left(\rho_{\mu} \rho_{\mu}\right)$. But it is easily shown that the introduction of a regulator in his calculations makes the additional term $\Gamma$ vanish. Indeed, we have

$$
\left(K_{\lambda \lambda}\right)_{R}=(2 \pi)^{-3} \Sigma_{i} c_{i} \int d^{4} k \delta\left(k_{\lambda} k_{\lambda}+M_{i}{ }^{2}\right)\left(\frac{p_{\lambda} p_{\lambda}-2 M_{i}{ }^{2}}{p_{\lambda} p_{\lambda}-2 \kappa_{\lambda} p_{\lambda}}-1\right) .
$$

It follows for a time like vector $\left(p_{\lambda}=o, i p_{0}\right)$ :

$$
\begin{aligned}
&\left(K_{\lambda \lambda}\right)_{R}=p_{0}{ }^{2}(2 \pi)^{-3} \Sigma_{i} c_{i} \int_{0}^{\infty} \frac{d k k^{2}}{\Omega_{i}(k)} \frac{2 \Omega_{i}{ }^{2}-M_{i}{ }^{2}}{\Omega_{i}{ }^{2}\left(p_{0}{ }^{2}-4 \Omega_{i}^{2}\right)} \\
&-2(2 \pi)^{-3} \Sigma_{i} c_{i} \int_{0}^{\infty} \frac{k^{2} d k}{\Omega_{i}} \quad\left[\Omega_{i}=\left(k^{2}+M_{i}^{2}\right)^{\frac{1}{2}}\right] .
\end{aligned}
$$

It is this second term that destroys covariance and gauge invariance; but since it can be written as

$$
-\left(1 /(2 \pi)^{3}\right) \sum_{i} c_{i}\left(K^{2}-\frac{1}{2} M_{i}^{2}\right)_{K \rightarrow \infty}
$$

we see that it vanishes on account of the two conditions (I, Ia). respect to $\alpha$ and $\beta$ ) with

$$
\begin{gathered}
K_{\mu \nu}(p)=\frac{\pi^{2}}{4(2 \pi)^{4}} \iint d \alpha d \beta(\epsilon(\alpha)+\epsilon(\beta)) \frac{\epsilon(\alpha+\beta)}{(\alpha+\beta)^{2}} \\
\times \exp \left[i \frac{\alpha \beta}{\alpha+\beta} p_{\lambda} p_{\lambda}+i(\alpha+\beta) m^{2}\right]\left\{\frac{-2 \alpha \beta}{(\alpha+\beta)^{2}} p_{\mu} p_{\nu}\right. \\
\left.+\delta_{\mu \nu}\left(\frac{\alpha \beta}{(\alpha+\beta)^{2}} p_{\lambda} p_{\lambda}-m^{2}-\frac{i}{\alpha+\beta}\right)\right\} .
\end{gathered}
$$

It is at this step that our regularization device comes into play, replacing $\exp \left[i(\alpha+\beta) m^{2}\right]$ by $R(\alpha+\beta)$, according to (9) and (11), and $m^{2} \exp \left[i(\alpha+\beta) m^{2}\right]$ by $(1 / i) R^{\prime}(\alpha+\beta) .{ }^{17}$ The regularized Eq. (42) reads then:

$$
\begin{aligned}
K_{R(\mu \nu)}= & \frac{\pi^{2}}{4(2 \pi)^{4}} \iint d \alpha d \beta(\epsilon(\alpha)+\epsilon(\beta)) \frac{\epsilon(\alpha+\beta)}{(\alpha+\beta)^{2}} \\
& \times \exp \left(i \frac{\alpha \beta}{\alpha+\beta} p_{\lambda} p_{\lambda}\right)\left\{\frac{2 \alpha \beta}{(\alpha+\beta)^{2}} R(\alpha+\beta)\right. \\
& \times\left(-p_{\mu} p_{\nu}+\delta_{\mu \nu} p_{\lambda} p_{\lambda}\right)-\delta_{\mu \nu}\left[\frac{\alpha \beta}{(\alpha+\beta)^{2}} p_{\lambda} p_{\lambda} R(\alpha+\beta)\right. \\
& \left.\left.+\frac{R(\alpha+\beta)}{\alpha+\beta}-i R^{\prime}(\alpha+\beta)\right]\right\} .
\end{aligned}
$$

Expressed in terms of the variables

which give

$$
\begin{aligned}
& z=\alpha+\beta \\
& y=1 / z(\alpha-\beta),
\end{aligned}
$$

$$
\epsilon(\alpha)+\epsilon(\beta)=2 \epsilon(z) \text { for }|y| \leq 1,
$$

and 0 elsewhere; and

$K_{R(\mu \nu)}$ reads:

$$
d \alpha d \beta=\frac{1}{2}|z| d z d y
$$

$$
\begin{aligned}
K_{R(\mu \nu)}= & \frac{\pi^{2}}{4(2 \pi)^{4}} \int_{-1}^{+1} d y \int_{-\infty}^{+\infty} \frac{d z}{z} \epsilon(z) \exp \left[i-\left(1-y^{2}\right) p_{\lambda} p_{\lambda}\right] \\
& \times\left\{R(z) \frac{1-y^{2}}{2}\left(-p_{\mu} p_{\nu}+\delta_{\mu \nu} p_{\lambda} p_{\lambda}\right)\right. \\
& \left.-\delta_{\mu \nu}\left[R(z) \frac{1-y^{2}}{4} p_{\lambda} p_{\lambda}+\frac{i}{z} R(z)-i R^{\prime}(z)\right]\right\} .
\end{aligned}
$$

${ }^{17}$ It may perhaps be helpful to show how a factorized regulator destroys gauge invariance. Taking a discrete spectrum of auxiliary masses, we have:

$$
\begin{aligned}
\frac{\partial K_{R(\mu \nu)}}{\partial x_{\nu}} & =\sum_{i j} c_{i} c_{j}\left\{\frac{\partial \Delta_{i}}{\partial x_{\mu}} \cdot M_{j}{ }^{2} \Delta_{j}^{(1)}+M_{i}{ }^{2} \Delta_{i} \frac{\partial \Delta_{j}^{(1)}}{\partial x_{\mu}}\right. \\
\left.-\frac{\partial}{\partial x_{\mu}}\left(M_{i} M_{j} \Delta_{i} \Delta_{j}^{(1)}\right)-\delta(x) \frac{\partial \Delta_{j}^{(1)}}{\partial x_{\mu}}\right\} & \\
& =\sum_{i j} c_{i} c_{j}\left(M_{j}-M_{i}\right)\left\{M_{i} \frac{\partial \Delta_{i}}{\partial x_{\mu}} \cdot \Delta_{j}^{(1)}-M_{i} \Delta_{i} \frac{\partial \Delta_{j}^{(1)}}{\partial x_{\mu}}\right\}
\end{aligned}
$$

which never vanishes identically. 
The contribution of the last term in the bracket may . be written as

$$
\begin{aligned}
& \frac{\pi^{2}}{4(2 \pi)^{4}} \delta_{\mu \nu} \int_{-1}^{+1} d y \int_{-\infty}^{+\infty} d z \epsilon(z) \\
& \times \frac{d}{d z}\left\{\frac{R(z)}{z} i \exp \left[\begin{array}{c}
z \\
i-\left(1-y^{2}\right) p_{\lambda} p_{\lambda} \\
4
\end{array}\right]\right\} \\
& =\frac{-2 i \pi^{2}}{4(2 \pi)^{4}} \delta_{\mu \nu} \int_{-1}^{+1} d y\left(\frac{R(z)}{z}\right)_{z=0}=\frac{-i \pi^{2}}{(2 \pi)^{4}} R^{\prime}(0) .
\end{aligned}
$$

According to our regularization condition $\left(\mathrm{I}^{\prime \prime} \mathrm{a}\right)$ this term is equal to zero and we are therefore left with an expression that has the required form (compare (35) and (36)):

$$
K_{R(\mu \nu)}(p)=K_{1}\left(p_{\lambda} p_{\lambda}\right)\left\{p_{\mu} p_{\nu}-\delta_{\mu \nu} p_{\lambda} p_{\lambda}\right\}
$$

where $K_{1}$ is given by:

$$
\begin{aligned}
K_{1}\left(p_{\lambda} p_{\lambda}\right)=\frac{-\pi^{2}}{4(2 \pi)^{4}} \int_{-\infty}^{+\infty} \frac{d z}{z} \epsilon(z) R(z) \\
\quad \times \int_{-1}^{+1} d y \frac{1-y^{2}}{2} \exp \left[\underset{i-}{i}\left(1-y^{2}\right) p_{\lambda} p_{\lambda}\right] .
\end{aligned}
$$

The first term in the expansion of (45) in powers of $p_{\lambda} p_{\lambda}$,

$$
K_{1}\left(p_{\lambda} p_{\lambda}\right)=K_{1}^{(0)}+p_{\lambda} p_{\lambda} K_{1}{ }^{(1)}+\cdots
$$

gives the charge renormalization (compare (37)):

$$
\delta e=4 e^{2} K_{1}{ }^{(0)}=\frac{-1}{3} \frac{\alpha}{2 \pi} \int_{-\infty}^{+\infty} \frac{d z}{z} \epsilon(z) R(z) .
$$

This may again be expressed, according to (10) and $\left(10^{\prime}\right)$, as

$$
\delta e=\frac{\alpha}{3 \pi} \int_{-\infty}^{+\infty} d \kappa \rho(\kappa) \log |\kappa|
$$

The connection with Wentzel's result for the photon self-energy is now most easily established. Since the field of a light wave is not connected with any current, $\left\langle j_{\mu}(x)\right\rangle_{\text {ind }}$ vanishes according to (37), unless $K_{\mu \nu}$ has not the required form (35), (36). From (43) and (44) it is made clear that in this case the induced current is given by

$$
\left\langle j_{\mu}(x)\right\rangle_{\mathrm{ind}}=-4 e^{2} \cdot \frac{-i \pi^{2}}{(2 \pi)^{4}} R^{\prime}(0) A_{\mu}{ }^{\mathrm{rad}}(x) .
$$

The photon self-energy, defined as

becomes thus:

$$
H_{\mathrm{self}}^{(\mathrm{ph})}=-\frac{1}{2} \int d^{3} x\left[\left\langle j_{\mu}(x)\right\rangle_{\mathrm{ind}} A_{\mu}^{\mathrm{rad}}(x)\right]_{1 \mathrm{ph}-0 \mathrm{ph}}
$$

$$
H_{\mathrm{self}}^{(\mathrm{ph})}=\frac{e^{2}}{8 \pi^{2}}\left(-i R^{\prime}(0)\right) \int d^{3} x\left[A_{\mu}^{\mathrm{rad}}(x) \cdot A_{\mu}^{\mathrm{rad}}(x)\right]_{1 \mathrm{ph}-0 \mathrm{ph}} .
$$

Since $R^{\prime}(0)$ is zero, the regularized photon self-energy vanishes, as it should; without regularization $R^{\prime}(0)$ $=i m^{2}$, which gives, together with

$$
\int d^{3} x\left[A_{\mu}(x) \cdot A_{\mu}(x)\right]_{1 \mathrm{ph}-0 \mathrm{ph}}=1 /|k|
$$

for a photon of momentum $k$

$$
H_{\text {self }}^{(\rho)}=\alpha / 2 \pi \cdot m^{2} /|k|
$$

which is exactly Wentzel's result.

\section{ADDITIONAL REMARKS}

(A) In $\left(38^{\prime}\right)$ we may be tempted to omit the term

$$
I(p)=\int d^{4} k \frac{\delta\left(k_{\lambda} k_{\lambda}+m^{2}\right)}{\left(k_{\lambda}-p_{\lambda}\right)^{2}+m^{2}}\left(k_{\lambda} k_{\lambda}+m^{2}\right)
$$

which means putting $\boldsymbol{\Delta}(x)\left(-\square^{2} \Delta^{(1)}(x)+m^{2} \Delta^{(1)}(x)\right)$ equal to zero, or its regularized counterpart:

$$
\int d \kappa \rho(\kappa) \Delta(x ; \kappa)\left(-\square^{2} \Delta^{(1)}(x ; \kappa)+\kappa \Delta^{(1)}(x ; \kappa)\right)=0 .
$$

An evaluation of $I_{R}(\rho)$ along the lines of the above calculations yields:

$$
\begin{aligned}
I_{R}(p)= & \sim \int_{-1}^{+1} d y \int_{-\infty}^{+\infty} \frac{d z}{z} \epsilon(z) \exp \left[i-\left(1-y^{2}\right) p_{\lambda} p_{\lambda}\right] \\
& \times\left[\left(\frac{-2}{z}+i \frac{\left.1+y^{2}\right]}{4} p_{\lambda} p_{\lambda}\right) R(z)+R^{\prime}(z)\right] \\
& +i p_{\lambda} p_{\lambda} \int_{1}^{\infty} d y y \int_{-\infty}^{+\infty} \frac{d z}{z} \epsilon(z) R(z) \\
& \times \exp \left[i-\left(1-y^{2}\right) p_{\lambda} p_{\lambda}\right] \\
= & \int_{-1}^{+1} d y \int_{-\infty}^{+\infty} d z \epsilon(z) \frac{d}{d z}\left\{\frac{R(z)}{z} \exp \left[i_{-}^{z}\left(1-y^{2}\right) p_{\lambda} p_{\lambda}\right]\right\} \\
& +\int_{-\infty}^{+\infty} \frac{d z}{z^{2}} \epsilon(z) R(z) \int_{-1}^{+1} d y \\
& \times \frac{d}{d y}\left\{y\left(1-\exp \left[-\frac{i z}{4}\left(1-y^{2}\right) p_{\lambda} p_{\lambda}\right]\right)\right\} \\
& =2 R^{\prime}(0)+0=0 \text { on account of }\left(\mathrm{II}^{\prime \prime} \mathrm{a}\right)
\end{aligned}
$$

Thus it may be seen that the identity (47) holds only because of our stronger regularization condition. It is of special interest to mention that it holds for a zero vector $\left(p_{\lambda} p_{\lambda}=0\right)$ only if the limit $p_{\lambda} p_{\lambda} \rightarrow 0$ is carried through after the integration. Putting $p_{\lambda} p_{\lambda}=0$ in $I(\rho)$, (48), from the very beginning gives rise to an additional term

$$
\sim \int_{-\infty}^{+\infty}\left(d z / z^{2}\right) \epsilon(z) R(z) .
$$

In consequence of this the non-regularized expression $I(0)$ becomes then infinite, whereas $I_{R}(0)$ is zero only 
if we put:

$$
\int\left(d z / z^{2}\right) \epsilon(z) R(z)=0
$$

or

$$
\int d \kappa \rho(\kappa) \kappa \log |\kappa|=0
$$

Thus Wentzel's result may as well have been infinite. From a physical point of view it appears, however, more natural to consider the case $\rho_{\lambda} \rho_{\lambda}=0$ as a limiting case of a non-zero vector, than to introduce the new condition (49) or $\left(49^{\prime}\right) .^{18}$

(B) At this point we shall briefly consider the case of the electron self-energy. In terms of $\boldsymbol{\Delta}(k), \Delta^{(1)}(k)$, $\bar{D}(k), D^{(1)}(k)$ the operator $\Delta m$ reads:

$$
\begin{aligned}
\Delta m & =\frac{\alpha}{2 \pi^{2}} \int d^{4} k(i \gamma k+m) \\
& \times\left(\frac{\delta\left(k_{\lambda} k_{\lambda}\right)}{\left(k_{\lambda}+q_{\lambda}\right)^{2}+m^{2}}+\frac{\delta\left(\left(k_{\lambda}+q_{\lambda}\right)^{2}+m^{2}\right)}{k_{\lambda} k_{\lambda}}\right)\left(q_{v} q_{\lambda}=-m^{2}\right) .
\end{aligned}
$$

This expression is readily transformed into

$$
\Delta m=\frac{m \alpha}{8 \pi} \int_{-\infty}^{+\infty} \frac{d z}{z} \epsilon(z) \int_{-1}^{+1} d y \frac{3-y}{2} \exp \left[i z\left(\frac{1-y}{2}\right)^{2}\right] .
$$

Regularization of both $D$ - and $\Delta$-functions without factorization corresponds to the introduction of a regulator $R(z) . R(z)$ here reduces to 1 in the case of non-regularization, in contrast to the definition of $R(z)$ previously used; in the case considered here, the expression to be regularized contains both $D$ - and $\Delta$ - functions, and $\kappa$ takes the signification of an additional contribution to the square of the mass; the mass attributed to $D$ is therefore $\kappa^{\frac{1}{2}}$, to $\Delta$ however $\left.\left(m^{2}+\kappa\right)^{\frac{1}{2}}\right)$. $\Delta m$ shall now be written as

$$
\begin{array}{r}
\Delta m=\frac{m \alpha}{16 \pi} \int_{-1}^{+1} d y(3-y) \int_{z}^{d z} \epsilon(z)\left(\exp \left[i z\left(\frac{1-y}{2}\right)^{2}\right]-e^{i z}\right) \\
+\frac{m \alpha}{16 \pi} \int_{z} \frac{d z}{z}(z) e^{i z} R(z) \int_{-1}^{+1} d y(3-y) .
\end{array}
$$

Since the first integral converges, no regulator is introduced. With the help of the formula

$$
\log |a|=-\frac{1}{2} \int_{-\infty}^{+\infty}(d z / z) \epsilon(z)\left(e^{i a z}-e^{i z}\right)
$$

we have at once:

$$
-(m \alpha / 8 \pi) \int_{-1}^{+1} d y(3-y) \log \left(\frac{1-y}{2}\right)^{2}=5 \cdot(m \alpha / 4 \pi) .
$$

${ }^{18}$ The situation is exactly the same in the case of the evaluation of the trace $K_{\lambda \lambda}$ by elementary $K$-space integrations (compare footnote (16)). The constant $\Gamma$ vanishes only if the additional assumption (which corresponds to $\left(49^{\prime}\right)$ )

$$
\Sigma_{i} c_{i} M_{i}^{2} \log M_{i}=0
$$

is made, unless this particular case is again considered as the limiting case of a non-zero vector.
If, in the second, divergent integral we introduce the special regulator

$$
R(z)=1-\exp \left[i z\left(\frac{1}{\gamma \omega_{0}}-1\right)\right]
$$

(which satisfies $\left(\mathrm{I}^{\prime \prime}\right): R(0)=0$, but not $R^{\prime}(0)=0$ ), we obtain

$$
-3 \cdot \frac{m \alpha}{8 \pi} \int_{z}^{d z} \epsilon(z)\left(\exp \left(i z \frac{1}{\gamma \omega_{0}}\right)-e^{i z}\right)=\frac{6 m \alpha}{8 \pi} \log \left|\frac{1}{\gamma \omega_{0}}\right|
$$

and thus

$$
\Delta m=3 m \alpha / 2 \pi\left(\frac{1}{2} \log \left|1 / \gamma \omega_{0}\right|+\frac{5}{6}\right),
$$

which is Schwinger's result (SII, Eq. (3.97)).

An alternative possibility is to regularize the photon$D$-functions alone, as was done by Feynman. ${ }^{7}$ In this case it is more convenient to introduce a regulator $\rho(\kappa)$; $(\Delta m)_{R}$ may then be written as

and

$$
(\Delta m)_{R}=\int d \kappa \rho(\kappa) \Delta m(\kappa)
$$

$$
\begin{aligned}
& \Delta m(\kappa)=\frac{m \alpha}{16 \pi} \int_{-1}^{+1} d y(3-y) \int_{z}^{d z} \epsilon(z) \\
& \times \exp \left[i z\left(\frac{\kappa}{2}(1+y)+\left(\frac{1-y}{2}\right)^{2}\right)\right] .
\end{aligned}
$$

( $\kappa$ is a dimensionless mass parameter, i.e., $m(\kappa)^{\frac{1}{2}}$ is the auxiliary mass.) Taking into account condition $\left(\mathrm{I}^{\prime}\right)$, we can omit all terms independent of $\kappa$; it follows therefore that

$$
\begin{aligned}
& \Delta m(\kappa)=\frac{-m \alpha}{8 \pi} \int_{-1}^{+1} d y(3-y) \log \left(\frac{\kappa}{2}(1+y)+\left(\frac{1-y}{2}\right)^{2}\right) \\
& =\frac{-m \alpha}{8 \pi} \int_{-1}^{+1} d y(y-5)(y-1) \frac{\kappa+(y-1)}{2 \kappa(1+y)+(1-y)^{2}} \\
& =\frac{-m \alpha}{8 \pi} F(\kappa) \\
& F(\kappa)= \begin{cases}-10+4 \pi(\kappa)^{\frac{1}{2}}+0(\kappa) & \kappa \ll 1 \\
6 \log \kappa-7+0\left(\frac{1}{\kappa}-\log \kappa\right) & \kappa \gg 1 .\end{cases}
\end{aligned}
$$

With the help of one auxiliary mass:

$$
\rho(\kappa)=\delta(\kappa)-\delta\left(\kappa-\frac{M^{2}}{m^{2}}\right)
$$

which is sufficient to satisfy $\left(\mathrm{I}^{\prime}\right)$, we obtain then

$$
\Delta m=\frac{3 m \alpha}{2 \pi}\left(\log \frac{M}{m}+\frac{1}{4}\right)
$$

which is Feynman's result. 


\section{§5. THE MAGNETIC MOMENT OF THE ELECTRON}

The $e^{2}$ radiative correction $\Delta j_{\mu}$ to the current, as given by (31) may be written as

$$
\begin{aligned}
\Delta j_{\mu}(p)=F\left(p_{\lambda} p_{\lambda}\right) \cdot \frac{1}{(2 \pi)^{4}} \int d^{4} q \bar{u}(p+q) \gamma^{\mu} u(q) \\
+G\left(p_{\lambda} p_{\lambda}\right) p_{\nu} \frac{1}{(2 \pi)^{4}} \int d^{4} q \bar{u}(p+q) \gamma^{[\mu \nu]} u(q),
\end{aligned}
$$

here $u(q), \bar{u}\left(q^{\prime}\right)$ are the Fourier-amplitudes of $\psi$ and $\bar{\psi}$ respectively, and $\gamma^{[\mu \nu]}=\frac{1}{2}\left(\gamma^{\mu} \gamma^{\nu}-\gamma^{\nu} \gamma^{\mu}\right)$.

For small values of $p, F$ and $G$ may be developed in powers of $p_{\lambda} p_{\lambda}$ (note that $p$ is the difference in momentum ascribed to $\bar{\psi}$ and $\psi$, respectively). Whereas $F(0)$ describes again a charge renormalization, the term corresponding to $G(0)$ exhibits an extra current, which, in $x$-space, has the more familiar form (32), and describes the radiative correction to the electron's magnetic moment in a homogeneous external field.

From the well-known decomposition of the current due to Gordon ${ }^{19}$

$$
\begin{array}{r}
i e \bar{u}(p+q) \gamma^{\mu} u(q)=e / 2 m\left\{\left(p_{\mu}+2 q_{\mu}\right) \bar{u}(p+q) u(q)\right. \\
\left.-p_{\lambda} \bar{u}(p+q) \gamma^{[\mu \lambda]} u(q)\right\}
\end{array}
$$

it is seen that the radiative correction to the magnetic moment may be expressed in terms of an anomalous $g$-factor

$$
\Delta g=-(4 m / e) G(0),
$$

since the unperturbed electron is characterized by $g=2$. The relevant terms of (31) (containing $G\left(p_{\lambda} p_{\lambda}\right)$ ) can be written as follows:

$$
\begin{array}{r}
-\left(i e^{3} / 2\right) \iint d^{4} \xi d^{4} \eta \bar{\psi}(x+\xi) \gamma^{\alpha}\left[\bar{D}(\xi-\eta) \bar{S}(\xi) \gamma^{\mu} S^{(1)}(-\eta)\right. \\
+\bar{D}(\xi-\eta) S^{(1)}(\xi) \gamma^{\mu} \bar{S}(-\eta) \\
\left.+D^{(1)}(\xi-\eta) \bar{S}(\xi) \gamma^{\mu} \bar{S}(-\eta)\right] \gamma^{\alpha} \psi(x+\eta)
\end{array}
$$

or, in momentum space (writing $q^{\prime}$ for $p+q$ ),

$$
\begin{gathered}
\frac{i e^{3}}{(2 \pi)^{3}} \bar{u}\left(q^{\prime}\right) \int d^{4} k \Omega_{\mu}(k)\left[\bar{D}(k) \Delta(k-q) \Delta^{(1)}\left(k-q^{\prime}\right)\right. \\
+\bar{D}(k) \Delta^{(1)}(k-q) \Delta\left(k-q^{\prime}\right) \\
\left.+D^{(1)}(k) \Delta(k-q) \Delta\left(k-q^{\prime}\right)\right] u(q)
\end{gathered}
$$

where

$$
\Omega_{\mu}(k)=2 i k_{\mu}(i \gamma \cdot k-m)-2 i\left(q_{\mu}{ }^{\prime}+q_{\mu}\right)(i \gamma \cdot k)+\sim \gamma^{\mu} .
$$

Let us introduce

$$
\begin{gathered}
T_{\sigma \tau}=\int d^{4} k k_{\sigma} k_{\tau}[D] \\
V_{\sigma}=\int d^{4} k k_{\sigma}[D] .
\end{gathered}
$$

\footnotetext{
${ }^{19}$ Compare Handbuch der Physik XXIV/I. 238.
}

( $[D]$ is the bracket in (53).) Since $[D]$ depends on the two parameters $q$ and $q^{\prime}$, or on

$$
p=q^{\prime}-q \text { and } Q=q^{\prime}+q,
$$

$T_{\sigma \tau}$ and $V_{\sigma}$ may be written as:

$$
\begin{aligned}
T_{\sigma \tau} & =\delta_{\sigma \tau} I_{0}+p_{\sigma} p_{\tau} I_{1}+Q_{\sigma} Q_{\tau} I_{2} \\
V_{\sigma} & =Q_{\sigma} J_{2} .
\end{aligned}
$$

All invariants thus introduced are still to be considered as functions of $p_{\lambda} p_{\lambda}$ (the invariant $Q_{\lambda} Q_{\lambda}$ reduces to the former by $\left.Q_{\lambda} Q_{\lambda}=-\left(4 m^{2}+p_{\lambda} p_{\lambda}\right)\right)$. With the help of (51) and the relations:

$$
\bar{u}\left(q^{\prime}\right) \gamma \cdot Q u(q)=2 i m \bar{u}\left(q^{\prime}\right) u(q), \quad \bar{u}\left(q^{\prime}\right) \gamma \cdot p u(q)=0
$$

we obtain from (54) and (55):

$$
\frac{i e^{3}}{(2 \pi)^{3}}(-2 i m)\left(2 I_{2}(p)-J_{2}(p)\right) p_{\lambda} \bar{u}(p+q) \gamma^{[\mu \lambda]} u(q)+\sim \gamma \mu
$$

thus yielding

$$
G\left(p_{\lambda} p_{\lambda}\right)=\frac{2 m e^{3}}{(2 \pi)^{3}}\left(2 I_{2}(p)-J_{2}(p)\right)
$$

and, according to (52):

$$
\begin{gathered}
\left(\alpha=e^{2} / 4 \pi=1 / 137\right) \\
\Delta g=-4 m^{2} / \pi\left(2 I_{2}(0)-J_{2}(0)\right)(\alpha / \pi) .
\end{gathered}
$$

The computation of the integrals involved in $I_{2}$ and $J_{2}$ may be carried through in different ways. A regularization device which guarantees the convergence of the expressions (54) for $T_{\sigma \tau}$ and $V_{\sigma}$ allows the introduction of a special coordinate system, characterized by in which

$$
p=0, \quad Q=(o, 2 \mathrm{im})
$$

$$
I_{2}(0)=1 / 4 m^{2}\left(T_{11}-T_{44}\right), \quad J_{2}(0)=(1 / 2 m) V_{4} .
$$

The most convenient regularization method is that which regularizes $[D]$ as a whole:

$$
([D])_{R}=\sum_{i} c_{i}[D]_{i}
$$

(according to (9))..$^{20}$ The evaluation of Eq. (57) in $k$-space is elementary. With the notations

$x=k / m, \quad \mu_{i}=M_{i} / m, \quad \Omega_{i}=\left(x^{2}+\mu_{i}^{2}\right)^{\frac{1}{2}}, \quad \mathbf{\Omega}_{i}=\left(1+x^{2}+\mu_{i}^{2}\right)^{\frac{1}{2}}$ we are led to:

$$
\left(I_{2}(0)\right)_{R}=\frac{\pi}{4 m^{2}} \int_{0}^{\infty} x^{2} d x \sum_{i} c_{i}\left\{\frac{\Omega_{i}{ }^{2}+\frac{1}{3} x^{2}}{\Omega_{i}{ }^{3}}-\frac{2+\Omega_{i}{ }^{2}+\frac{1}{3} x^{2}}{\mathbf{\Omega}_{i}{ }^{3}}\right\}
$$

$\left(J_{2}(0)\right)_{R}=\frac{\pi}{2 m^{2}} \int_{0}^{\infty} x^{2} d x \sum_{i} c_{i} \frac{1}{\Omega_{i}{ }^{2} \boldsymbol{\Omega}_{i}{ }^{3}}$.

Equation (58a) converges without regularization and yields

$$
J_{2}(0)=\pi / 2 m^{2}
$$

a value which remains unaltered under regularization,

${ }^{20} \mathrm{~A}$ control calculation, carried through with a regulator affecting only the electron $\Delta$-functions in $[D]$ gave exactly the same results. 
since all terms $i>0$ vanish at least as $c_{i} / M_{i}{ }^{2}$ (compare (6)). This is not the case, however, for (58), which becomes

$$
\left(I_{2}(0)\right)_{R}=\frac{\pi}{12 m^{2}}\left(2 \sum_{i} c_{i}-\frac{3}{2} \sum_{(i>0)}^{\prime} c_{i}\right) .
$$

The expression (58) proves thus to be convergent without regularization; we need only put $c_{0}=1, c,(i>0)=0$, to obtain

$$
I_{2}(0)=\pi / 6 m^{2}
$$

whereas the properly regularized expression yields

Therefore

$$
\left(I_{2}(0)\right)_{R}=\pi / 8 m^{2} \text {. }
$$

whereas

$$
2 I_{2}(0)-J_{2}(0)=-\pi / 6 m^{2}
$$

$$
2\left(I_{2}(0)\right)_{R}-J_{2}(0)=-\pi / 4 m^{2} .
$$

The regularized value, introduced in (56), gives

$$
\Delta g=\alpha / \pi
$$

in agreement with Schwinger's result. ${ }^{21}$

From this example it should be made clear how cautious one should be in handling divergent "covariant" expressions. Indeed, any decomposition, as done in (55), is by no means "covariant," as long as the expressions under discussion has not been made convergent by some invariant regularization. Once this has been done, one may quietly enjoy of all the facilities presented by a properly chosen coordinate system, as was done in (57).

\section{ADDITIONAL REMARKS}

(A) The various possibilities of evaluating the integrals $I_{2}$ and $J_{2}$ arise from the different possibilities of rewriting the $[D]$-bracket in (54), (54a). It is easily seen from the definitions (40), (41) that for $q=q_{1}{ }^{\prime},[D]$ may be written as

$$
[D]=\int_{0}^{1} d u u \delta^{\prime \prime}\left(k_{\lambda} k_{\lambda}-2 k_{\lambda} q_{\lambda} u\right) .{ }^{22}
$$

The regularization of $[D]$, as a whole, as was done in the above calculations, consists then in replacing (60) by

$$
([D])_{R}=\int_{0}^{1} d u u \sum_{i} c_{i} \delta^{\prime \prime}\left(k_{\lambda} k_{\lambda}-2 k_{\lambda} q_{\lambda} u+M_{i}^{2}\right) .
$$

Equation (61) may now be introduced into (54):

$$
\begin{gathered}
\left(T_{\sigma \tau}\right)_{R}=\int_{0}^{1} d u u \int d^{4} k k_{\sigma} k_{\tau} \sum_{i} c_{i} \delta^{\prime \prime}\left(k_{\lambda} k_{\lambda}-2 k_{\lambda} q_{\lambda} u+M_{i}^{2}\right) \\
=\int_{0}^{1} d u u \int d^{4} k\left(k_{\sigma} k_{\tau}+u^{2} q_{\sigma} q_{\tau}\right) \\
\quad \times \sum_{i} c_{i} \delta^{\prime \prime}\left(k_{\lambda} k_{\lambda}+m^{2} u^{2}+M_{i}^{2}\right) .
\end{gathered}
$$

${ }^{21}$ J. Schwinger, Phys. Rev. 73, 416 (1948).

22 This method is due to Schwinger; (SIII).
Thus $I_{2}(0)$ is immediately isolated:

$$
\begin{aligned}
I_{2}(0) & =\frac{1}{4} \int_{0}^{1} d u u^{3} \sum_{i} c_{i} \int d^{4} k \delta^{\prime \prime}\left(k_{\lambda} k_{\lambda}+m^{2} u^{2}+M_{i}^{2}\right) \\
& =\frac{\pi}{4} \sum_{i} c_{i} \int_{0}^{1} d u u^{3} \frac{1}{m^{2} u^{2}+M_{i}^{2}}=\frac{\pi}{8 m^{2}}+0\left(\frac{c_{i}}{M_{i}^{2}}\right)_{i>0} .
\end{aligned}
$$

The use of a regulator appears to be superfluous in this case, but from a general point of view it is doubtful whether a transformation of variables as needed to obtain (62) can be justified a priori.

(B) $[D]$ may, according to Dyson, also be written as

$$
-\frac{1}{4} \operatorname{Re}\left(D_{c}(k) \Delta_{c}(k-q) \Delta_{c}\left(k-q^{\prime}\right)\right)
$$

where

$\Delta_{c}(k)=\Delta_{1}-2 i \Delta=2 \pi \delta_{+}\left(k^{2}+m^{2}\right), \quad\left(\right.$ and $\left.\quad D_{c}=D^{(1)}-2 i \bar{D}\right)$.

With the elementary (complex) $k$-space integration, however, one immediately falls back on the formula (58), (58a).

(C) Finally we illustrate the possibility of obtaining a quite arbitrary result by regularizing in a different way different terms of a form $F\left(\boldsymbol{\Delta}, \Delta^{(1)}\right)$. Let us just take, as an example, the bracket $[D]$, which for $q^{\prime}=q$ may be written as follows:

$$
[D]=\frac{\delta\left(k_{\lambda} k_{\lambda}\right)}{\left(2 k_{\lambda} q_{\lambda}\right)^{2}}-\frac{1}{k_{\lambda} k_{\lambda}} \delta^{\prime}\left(k_{\lambda} k_{\lambda}-2 k_{\lambda} q_{\lambda}\right)=\left[D_{1}\right]+\left[D_{2}\right] .
$$

We need now only note that if $R_{1}$ and $R_{2}$ are two regulators, the operation $R=\frac{1}{2}\left(R_{1}+R_{2}\right)$ is a regulator too, whereas $\bar{R}=\frac{1}{2}\left(R_{1}-R_{2}\right)$ is an operator, corresponding to a mass spectrum which satisfies (I, Ia), but contains only auxiliary masses $M_{i}$; we may now write

$$
R_{1}\left[D_{1}\right]+R_{2}\left[D_{2}\right]=R[D]+\bar{R}\left(\left[D_{1}\right]-\left[D_{2}\right]\right) .
$$

The additional terms depending on $\bar{R}$ are by no means zero in general, but depend on the structure of $\bar{R}$ which shall be characterized by a spectrum $M_{i}$ and coefficients $\gamma_{i}$. In evaluating $I_{2}(0)(54),(55)$, as an example, the additional terms due to $\bar{R}$ are:

$$
\pi / 4 m^{2}\left(\sum_{i} \gamma_{i} \mu_{i}^{2} \log \mu_{i}-\frac{2}{3}\right)
$$

an expression which is completely indeterminate.

\section{ACKNOWLEDGMENTS}

We have to acknowledge. Professor J. Schwinger, Dr. F. J. Dyson, Professor V. F. Weisskopf, Dr. S. T. $\mathrm{Ma}$, and Dr. K. M. Case for making their publications accessible to us prior to publication; Dr. G. Rayski for valuable help, and Professor E. C. G. Stueckelberg and Dr. G. Rivier for interesting discussions. 